\title{
INTENSIFICACIÓN AGRÍCOLA Y PÉRDIDA DE SERVICIOS AMBIENTALES EN EL PARTIDO DE AZUL (PROVINCIA DE BUENOS AIRES) ENTRE 2002-2011
}

\section{Agricultural intensification and loss of environmental services in the Azul county, Province of Buenos Aires (Argentina) between 2002-2011}

Patricia Vazquez

MSc. en Teledetección, Lic. en Diagnóstico y Gestión Ambiental CONICET / Universidad Nacional del Centro de la Provincia de Buenos Aires, Argentina patriciavazquez11@gmail.com

Laura Zulaica

Dra. en Geografía, Lic. en Diagnóstico y Gestión Ambiental CONICET / Universidad Nacional de Mar del Plata, Argentina laurazulaica@yahoo.com.ar

Artigo recebido em 21/03/2013 e aceito para publicação em 26/06/2013

RESUMEN: En las últimas décadas, la agricultura Argentina, especialmente en la ecorregión de las Pampas, ha experimentado una notable expansión basada en el desarrollo tecnológico. Este proceso permite aumentar la productividad pero incrementa los costos en términos de pérdida de servicios ambientales. El presente trabajo propone analizar la pérdida de algunos servicios de soporte y regulación en el período 2002-2011, en un área representativa de la ecorregión: el partido de Azul. Para ello, se utilizaron dos indicadores de sustentabilidad: conservación de la calidad de suelos y agua (CCSA) y conservación de la biodiversidad (CB). Previamente, se analizaron los cambios de uso del suelo mediante el procesamiento y clasificación de imágenes satelitales, entrevistas y campañas de campo. Los resultados obtenidos indican una intensificación y expansión agrícola $(13,79 \%)$, a expensas de la ganadería $(-5,24 \%)$ y de la superficie cubierta con agua en 2002. Dichos cambios se reflejan en la pérdida de servicios ambientales y se traducen en la estimación de los indicadores: el indicador de CCSA disminuyó 33,8\% y el de CB 16,03\%. Ante lo enunciado, se considera prioritario generar estrategias agroproductivas contemplando que los servicios que prestan los ecosistemas son indispensables para el sostenimiento del Partido y la región.

Palabras clave: agriculturización; sensores remotos; indicadores de sustentabilidad; conservación de recursos naturales.

ABSTRACT: In recent decades, Argentinean agriculture, especially in the Pampas ecoregion, has undergone a remarkable expansion based on technological development. This process allows increasing productivity but it increases costs in terms of loss of environmental services. This paper aims at analyzing the loss of some support and regulation services in the period 2002-2011, in a representative area of the ecoregion: Azul County. In order to conduct such analysis, two sustainability indicators were used: quality conservation of soil and water (QCSW) and biodiversity conservation (BC). Land use changes by processing and classifying satellite images, interviews, and field campaigns were previously analyzed. The results indicate an intensification and expansion of agriculture (13.79\%) to the detriment of livestock (-5.24\%) and of the surface covered with water in 2002. These changes are reflected in the loss of environmental services and result in the estimation of the indicators: QCSW indicator decreased by $33.8 \%$ and BC by $16.03 \%$. Thus, it is a priority to generate agroproductive strategies contemplating that services provided by ecosystems are essential for the maintenance of the County and the region.

Keywords: agriculturization; remote sensing; sustainability indicators; conservation of natural resources. 


\section{INTRODUCCIÓN}

En el territorio argentino y latinomericano, muchos ecosistemas que hasta hace apenas un par de décadas permanecieron bajo vegetación natural y fueron destinados a usos ganaderos y forestales muy extensivos, e incluso en algunos casos considerados como "áreas de desperdicio", están cediendo espacio para el avance de la frontera agropecuaria a tasas sin precedentes. El mejoramiento genético, la biotecnología, el control químico de plagas, los sistemas de labranza, irrigación y drenaje han progresado de forma notable y permiten responder rápidamente a las demandas del mercado internacional por alimentos y fibras, así como de su nuevo competidor, los biocombustibles (LATERRA et al., 2011).

En la ecorregión de las Pampas, $540.000 \mathrm{Km}^{2}$ (BURKART et al., 1999), ha tenido lugar un importante proceso de "agriculturización". Manuel-Navarrete y Gallopín (2007), concuerdan en que el proceso denominado "agriculturización", implica el uso creciente y continuo de las tierras para cultivos agrícolas en reemplazo de usos ganaderos o mixtos y, desde la perspectiva de la región pampeana, se lo vincula con los cambios tecnológicos, intensificación ganadera, expansión de la frontera agropecuaria hacia regiones extrapampeanas y tendencia al monocultivo de soja. Dicho proceso conduce a una simplificación estructural y funcional de los sistemas productivos que, si bien permite aumentar la productividad y rentabilidad actual, también aumenta los costos ambientales en términos de degradación del suelo, contaminación del agua y del aire, pérdida de diversidad biológica y deterioro de servicios ecológicos esenciales (VIGLIZZO, 2008).

Ahora bien, las concepciones más modernas en materia de gestión ambiental destacan la necesidad de valorar los bienes y servicios que ofrecen los ecosistemas, de asimilar la noción de agricultura multi-funcional y de incorporar el concepto de gestión del ambiente rural. Proyectadas territorialmente, estas nociones son básicas para instrumentar políticas y estrategias que ayuden a mejorar la calidad de vida de las personas que habitan distintas ecorregiones y ecosistemas de la Argentina (CARREÑO; VIGLIZZO, 2011). Por tanto, se debe aceptar que aunque un servicio ecológico no tenga un precio, tiene un valor que la sociedad debe aprender a apreciar.
El concepto de "servicios ecosistémicos o ambientales" es relativamente nuevo y su aplicación y desarrollo mantiene abierto hoy un gran debate respecto de la manera en que pueden afectar positiva o negativamente los ecosistemas y las comunidades (SEMARNAT, 2003).

Para establecer el posible origen del concepto se puede acudir a la definición "los ecosistemas han sido afectados por actividades como el cambio en el uso del suelo, la generación de desechos y el deterioro de los recursos naturales, causando la pérdida de sus funciones y servicios y reduciendo la riqueza natural global y el bienestar social" (MEA, 2005). En esta definición se habla expresamente de la reducción de funciones y servicios que proveían los ecosistemas, con lo que se da un giro importante en los conceptos de ecosistemas como algo cerrado y estable para permitir una mirada más dinámica flexible y adaptada a procesos más que a estados. Pero se abre además la posibilidad de ver los ecosistemas como proveedores de servicios para las sociedades con lo que el debate entre lo natural y lo social encuentra un punto de fusión bien interesante, ya que dicha división antes tajante, queda ahora borrada por la necesaria interrelación que crea el término o mejor el concepto de servicios ecosistémicos.

Dentro del marco conceptual introducido por el programa Millennium Ecosystem Assessment (MEA, 2003; 2005), los servicios del ecosistema pueden ser agrupados en cuatros categorías: de provisión, de regulación, culturales y de soporte.

De acuerdo con las fuentes citadas, los servicios de provisión -alimentos, fibras, agua y materias prima- son aquellos que generalmente resultan tangibles a los sentidos del hombre y poseen un valor de mercado. Los servicios de regulación -control de erosión, ciclado de nutrientes, purificación de agua, control de disturbios, regulación del clima y de las aguas- tienen, en cambio, un valor funcional que es invisible al ojo humano y no cotiza por tanto en el mercado. Su deterioro o escasez se manifiesta en problemas que en general se perciben tardíamente. Los servicios culturales -tales como patrimonio histórico, costumbres, lenguas, comidas, relaciones sociales- comienzan a ser valorados crecientemente en las sociedades organizadas, y valorizados en tér- 
minos económico-comerciales a través de actividades como el agroturismo, el eco-turismo, los servicios de recreación. Por último, los servicios de soporte -provisión de agua dulce, mantenimiento del hábitat, conservación de la biodiversidad, formación de suelos-, que tampoco cotizan en los mercados actuales, son también intangibles pero esenciales para sostener a los restantes servicios naturales.

Existen varios estudios sobre la ecorregión de las Pampas, a distintas escalas geográficas (potrero, establecimiento rural, cuenca, ecosistema, eco-región), que han demostrado que existen impactos directos e indirectos de factores tales como cambios en el uso del suelo, uso de insumos externos, aplicación de tecnología, pérdida de biodiversidad, fragmentación de hábitat (SOLBRIG; VIGLIZZO, 1999; VIGLIZZO et al., 2003; VAZQUEZ; ZULAICA, 2012; VAZQUEZ et al., 2012; entre otros).

Asimismo, estos factores tangibles e intangibles son afectados por otros factores indirectos. Dentro de esta categoría se incluyen las políticas socioeconómicas y ambientales, estrategias comerciales del sector privado, fluctuaciones del mercado, cambios demográficos y cambios tecnológicos (MEA, 2003).

En el marco enunciado y partiendo de estudios antecedentes (VAZQUEZ; ZULAICA, 2012; VAZQUEZ et al., 2012), el presente trabajo propone analizar la pérdida de algunos servicios ambientales de soporte y regulación en el período 2002-2011, en un área cuya diversidad ecosistémica caracteriza la ecorregión de las Pampas: el partido de Azul, provincia de Buenos Aires. Para ello, la investigación se vale del uso de indicadores de sustentabilidad (RUEDA, 1999; VARSAVSKY; FERNÁNDEZ DILLON, 2003) que permiten evaluar en qué medida los servicios ambientales de conservación de la biodiversidad y conservación de la calidad de suelos y aguas son afectados como consecuencia del proceso de agriculturización evidenciado en el área de estudio en el período considerado.

Frente al objetivo perseguido y teniendo en cuenta que cualquiera sea el método adoptado para alcanzar ese fin, es importante recordar que la valuación de los servicios ecosistémicos, o bien ambientales como se denominan en este trabajo, no constituye un objetivo en sí mismo, sino que es una herramienta más, destinada a orientar el proceso de la toma de decisiones (DAILY et al., 2000).

\section{Área de estudio}

En el partido de Azul $\left(6.615 \mathrm{Km}^{2}\right)$, inserto en la ecorregión de Las Pampas, se evidencia el proceso de "agriculturización", y por ende también son representativos los cambios generados en los servicios ambientales de soporte y regulación, que alteran la funcionalidad de los ecosistemas naturales y sus servicios asociados.

E1 Partido, posee 65.280 habitantes (INDEC, 2010) y se localiza en el centro de la Provincia de Buenos Aires (Figura 1). Los partidos limítrofes son: al norte, Las Flores y Tapalqué; al este, Rauch y Tandil; al oeste, Olavarría y; al sur, Benito Juárez. Azul se halla comunicado con todo el país a través de las rutas nacionales $\mathrm{N}^{\circ} 3$ y 226 , las provinciales $\mathrm{N}^{\circ} 51$ y 60. Dista $300 \mathrm{Km}$ de la Capital Federal, $240 \mathrm{Km}$ del Puerto de Quequén y $365 \mathrm{Km}$ de Bahía Blanca.

El clima regional es de tipo templado húmedo con influencia oceánica, inviernos suaves y veranos cortos y frescos. El régimen de precipitaciones presenta un promedio anual de $900 \mathrm{~mm}$ con una distribución normal y una mayor concentración en el período primavero-estival. Registra un exceso de las precipitaciones sobre la evapotranspiración durante los meses de invierno lo cual, junto a la escasa pendiente y la baja permeabilidad de los suelos en algunas zonas, genera frecuentes inundaciones. Durante el verano puede presentarse un leve déficit hídrico. Entre los factores adversos para la agricultura se encuentran también las heladas que, aunque rara vez se producen fuera de época, se caracterizan por su variabilidad. El período libre de heladas oscila entre 123 y 245 días, y no se registran años sin heladas (Centro Regional de Agrometeorología, FAA-UNICEN).

Desde el punto de vista fitogeográfico el área de estudio forma parte de la Provincia Pampeana descripta por Cabrera y Willink (1973). Los autores citados definen como tipo de vegetación dominante la estepa o pseudoestepa de gramíneas, que forman matas de $60 \mathrm{~cm}$ a $1 \mathrm{~m}$ de altura. 
Figura 1. Localización del partido de Azul (Provincia de Buenos Aires).

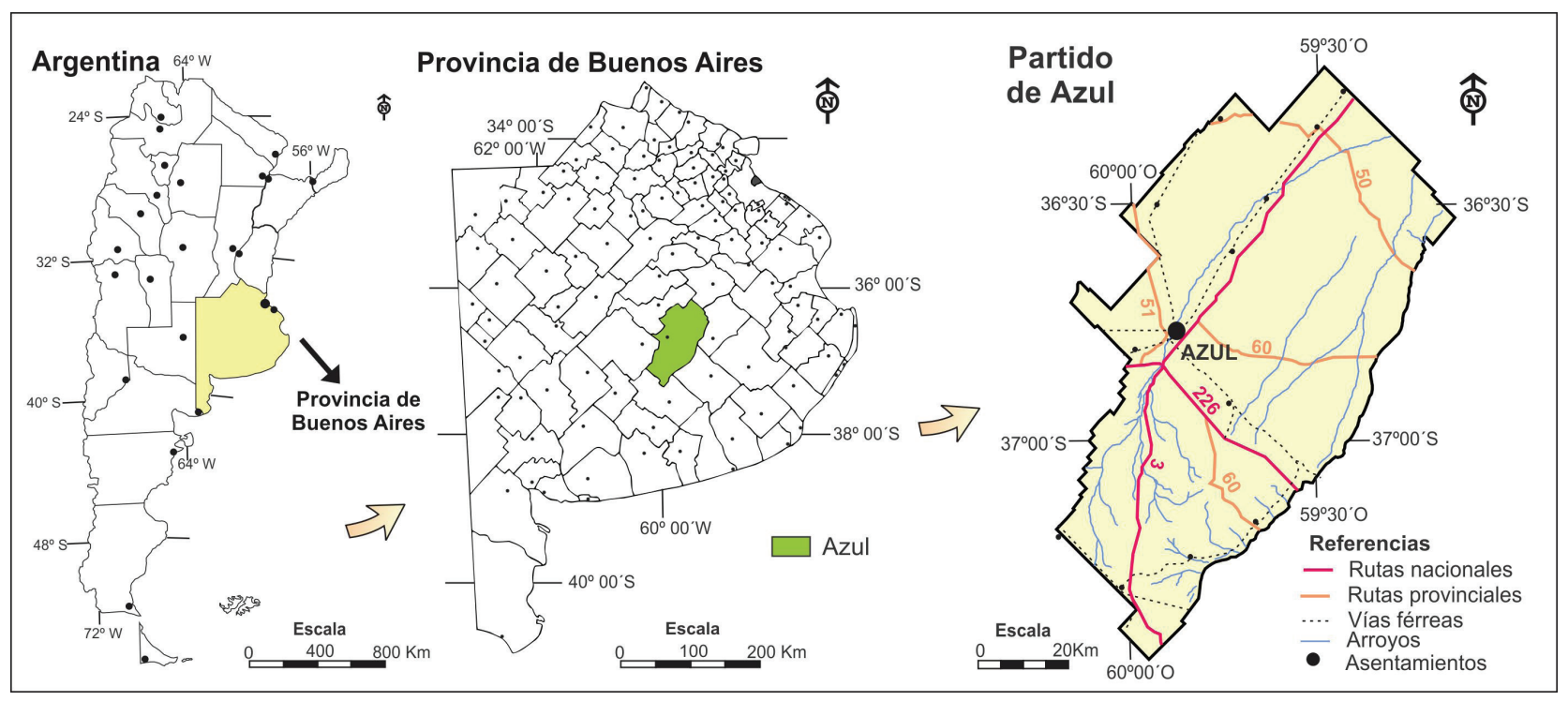

Fuente: Elaboración propia.

Tomando como base estudios antecedentes realizados en el partido de Tandil (SÁNCHEZ et al., 1999), se delimitaron tres compartimentos ecológicos en el partido de Azul denominados Serranías, Llanuras periserranas y Planicies deprimidas. De acuerdo con la descripción realizada, el sistema de las Serranías integra cerros, laderas serranas y lomadas altas. Además de numerosos afloramientos, las diferentes superficies geomórficas asocian suelos poco profundos en los que crecen diferentes comunidades de gramíneas y herbáceas. Los núcleos serranos auspician el desarrollo de una red hidrológica de alcance regional. Los diferentes bloques de cerros derivan en llanuras onduladas morfoestructuralmente influenciadas por el borde serrano y que constituyen el compartimento ecológico denominado Llanuras periserranas. Dichas llanuras contactan (en altitudes menores) con llanuras poco inclinadas y llanuras francamente chatas o deprimidas que en su conjunto conforman el compartimento que ha sido denominado Planicies deprimidas. En ambos tipos de formaciones de llanura la profundidad de los suelos se ve a veces interrumpida por una costra calcárea (tosca). Los pastizales constituían la fisonomía general de estas llanuras. Sin embargo las Llanuras periserranas y algunas Planicies deprimidas han sido extensivamente sustituidas por agroecosistemas.

\section{METODOLOGÍA}

Con la finalidad de evaluar la pérdida de servicios ambientales de soporte y regulación en el partido de Azul durante el período 2002-2011, se construyeron y aplicaron dos indicadores de sustentabilidad definidos en este trabajo: conservación de la calidad de suelos y agua (CCSA) y conservación de la biodiversidad (CB).

Los indicadores pueden ser definidos como variables dotadas de significados, derivados de su propia configuración científica, que reflejan en forma sintética un interés social por el ambiente posible de ser incluido coherentemente en el proceso de toma de decisiones (RUEDA, 1999). Proveen información en forma concisa y científicamente válida, que pueden ser fácilmente comprendidas y utilizadas. Por ello, son herramientas necesarias para evaluar el estado de un sistema y su evolución en el tiempo. También permiten identificar ineficiencias en las prácticas usuales, fijar prioridades para trabajos futuros, evaluar su efectividad, corregir desvíos y tendencias, medir mejoras e informar progresos. Es necesario para que sea posible que las políticas, los indicadores y los métodos de evaluación reflejen adecuadamente las variables locales y regionales (VARSAVSKY; FERNÁNDEZ DILLON, 2003). 
El indicador CCSA refiere a la conservación de los recursos relacionada específicamente con el uso de plaguicidas, mientras que el indicador $\mathrm{CB}$ refleja el estado de conservación del hábitat en un área determinada que depende de los procesos productivos impuestos sobre la biodiversidad. Para calcularlos, se tomaron como base estudios antecedentes (VAZQUEZ; ZULAICA, 2012; VAZQUEZ et al., 2012) y fundamentalmente el cálculo de dos indicadores obtenidos del Software Agro-Eco-Index (Riesgo de Contaminación por Plaguicidas, RCP y Riesgo de Intervención del Hábitat, RIH), desarrollados por Viglizzo (2003), con modificaciones realizadas a partir de los datos disponibles. Los indicadores utilizados adoptan un valor comprendido entre 0 y 1 que expresan la peor y mejor condición de conservación, respectivamente.

El cálculo de los indicadores demandó el conocimiento de información precisa del área de estudio referida al proceso de agriculturización y a los plaguicidas empleados en 2002 y 2010. Esto requirió del análisis de los cambios de uso del suelo en el período seleccionado a partir de sensores remotos y de la realización de entrevistas a informantes calificados.

A fin de verificar los cambios en los usos del suelo en el período para analizar el proceso de agriculturización, se utilizaron imágenes satelitales del área de estudio correspondientes a 2002 y 2011. Las imágenes se obtuvieron del sitio Web http://www.inpe. br/, página correspondiente al Instituto Nacional de Pesquisas Espaciais (INPE) del Ministerio de Ciencia e Tecnología de Brasil.

Se realizó una calibración radiométrica de las imágenes, se llevaron a la proyección UTM- Datum WGS-84 - Zona 21 Sur y se georreferenciaron (Software ENVI 4.5) utilizando como imagen base la provista por el recorte de un mosaico de imágenes (2135) del sensor ETM+, obtenido de la página Web Global Land Cover Facility, Earth Science Data Interface (http://www.landcover.org/).

Se realizaron luego los mosaicos correspondientes a cada año seleccionado y se creó un vector del partido de Azul, el cual permitió crear una máscara y aplicarla con el propósito de recortar el área correspondiente a dicho Partido. Posteriormente, se realizó una clasificación supervisada en la que se aplicó el Clasificador de Máxima Probabilidad (ENVI 4.5), que es el más complejo y el que demanda mayor volumen de cálculo. Sin embargo, es el más empleado en la teledetección, por su robustez y por su ajustarse con mayor rigor a la disposición general de los datos (CHUVIECO, 2007).

Una vez obtenida la imagen clasificada, se aplicó un filtro Median ( $3 * 3)$, técnica que permite mejorar el contraste espacial de la imagen (CHUVIECO, 2007). Se obtuvieron los estadísticos de las imágenes clasificadas, los píxeles por cada clase y se estimó la superficie de las mismas. Con todo lo anterior, se analizaron los cambios en los usos de suelo para así verificar el avance de las áreas agrícolas y calcular los indicadores seleccionados.

A fin de evaluar el servicio de CCSA, se utilizó la siguiente fórmula:

$$
C C S A=1-\frac{\left(\frac{1000}{D L 50}\left[\frac{K s p+R}{2}+K o c+T \quad 1 / 2\right] \times C \times S\right)}{10000000000}
$$

Donde: DL 50, es el promedio de la dosis letal de los principales plaguicidas utilizados, que determina la toxicidad de los compuestos; Ksp, es el promedio de la solubilidad en agua de los principales plaguicidas utilizados; $\mathbf{R}$, expresa la permeabilidad del suelo en las capas superficiales; Koc, es el promedio de coeficientes de adsorción de los compuestos por la fase orgánica del suelo; $\mathbf{T}^{1} / 2$, es el promedio de la vida media de los productos utilizados; $\mathbf{C}$, expresa la cantidad de producto aplicada por unidad de superficie; y $\mathbf{S}$, es la superficie total en la cual se aplica el producto (en este caso, corresponde a las áreas agrícolas del Partido obtenidas a partir de la clasificación supervisada de imágenes satelitales). Dado que el indicador se calculó considerando un espacio regional, la ecuación se ajustó dividiendo luego el resultado final, para evitar números extensos.

Para evaluar el servicio de CB, se consideraron las principales actividades desarrolladas en el Partido y se calculó el Riesgo de Parcial Intervención del Hábitat (RPIH). Mediante el uso de determinados coeficientes, el indicador compara la vegetación actual con la vegetación potencial del mismo, es decir la vegetación que se supone que habría si el hombre no hubiese intervenido en el proceso sucesional (VIGLIZZO, 2003). Los coeficientes utilizados para lograr esta comparación se presentan en la Tabla 1. 
Tabla 1. Coeficientes utilizados para estimar el Riesgo Parcial de Intervención del Hábitat.

\begin{tabular}{|l|}
\hline \multicolumn{1}{c|}{ Cantidad de especies } \\
\hline $\begin{array}{l}\text { Este es el coeficiente que se considera de mayor importancia relativa, y se asignan } 10 \text { puntos de impacto si existe un cambio } \\
\text { significativo (tanto pérdida como ganancia de especies) en la cantidad de especies debido a la intervención humana y } 0 \\
\text { puntos si no hay cambio. }\end{array}$ \\
\hline $\begin{array}{l}\text { Se asignan 7,5 puntos de impacto si una proporción significativa de las especies presentes en la vegetación actual son intro- } \\
\text { ducidas a un determinado ecosistema. Se parte del supuesto que la vegetación nativa, al haber coexistido con la fauna nativa, } \\
\text { tiene mayor capacidad de servirle de hábitat que la vegetación exótica. }\end{array}$ \\
\hline \multicolumn{1}{c|}{ Periodicidad } \\
\hline $\begin{array}{l}\text { Referida a la vegetación dominante. Si la vegetación potencial era perenne y es reemplazada por especies anuales (aún si } \\
\text { fueran nativas), se asignan al potrero correspondiente } 5 \text { puntos de impacto. Lo mismo sucede si la vegetación potencial era } \\
\text { mayoritariamente anual y pasa a ser perenne. }\end{array}$ \\
\hline $\begin{array}{l}\text { Se asume que una mayor cantidad de estratos se corresponde con una mayor disponibilidad de sitios para su utilización } \\
\text { como hábitats naturales. Por consiguiente, un cambio en este número determina un cambio en la capacidad de provisión de } \\
\text { refugio y alimento de los ecosistemas. Se asignan 2,5 puntos de impacto cuando esto ocurre. }\end{array}$ \\
\hline $\begin{array}{l}\text { Tiene un nivel de importancia relativa menor e incluye los cambios en la cantidad de sub-estratos dentro de alguno (o algu- } \\
\text { nos) de los estratos principales. }\end{array}$ \\
\hline \begin{tabular}{l} 
Organización en sub-estratos verticales \\
\hline
\end{tabular} \\
\hline
\end{tabular}

Elaboración propia.

Para obtener el valor del indicador de RPIH en el Partido, denominado así en este presente trabajo siguiendo los antecedentes de Vazquez y Zulaica (2012) y Vazquez et al. (2012), se suman los coeficientes obtenidos para cada actividad y se divide dicha sumatoria por 26 (para obtener un valor de cero a uno). El RPIH queda expresado como se muestra a continuación:

$$
\mathrm{RPIH}=\frac{\mathrm{Ce}+\mathrm{Or}+\mathrm{Pe}+\mathrm{Oev}+\mathrm{Osv}}{26}
$$

Donde: RPIH, es el indicador de riesgo parcial de intervención del hábitat; $\mathbf{C e}$, es el coeficiente relativo a la cantidad de especies; $\mathbf{O r}$, indica el coeficiente referido al origen; $\mathbf{P e}$, expresa el coeficiente de periodicidad; Oev, es el coeficiente de organización de estratos verticales; y Osv, corresponde al coeficiente de estratos subverticales.

Posteriormente, los valores obtenidos para cada actividad (RPIH) se multiplican por un coeficien- te de ponderación que expresa la superficie ocupada por cada actividad en el Partido. De esta manera, la sumatoria de los valores obtenidos expresa el riesgo de intervención del hábitat (RIH) definido por Viglizzo (2003), en tanto que el indicador CB, queda expresado de la siguiente forma:

$$
C B=1-\sum c p S u p A c * R P I H
$$

Donde: $\mathbf{C B}$, es el indicador de Conservación de la Biodiversidad; cpSupAc, es el coeficiente de ponderación que indica la proporción de la superficie del Partido ocupada por la actividad, obtenida a partir de la clasificación supervisada de imágenes satelitales; y RPIH, es el indicador parcial de riesgo de intervención del hábitat.

Con todo lo anterior, se calcularon ambos indicadores para 2002 y 2011 a partir de los datos aportados por la clasificación supervisada de las imágenes de satélite de esos años y corroboraciones en campo. 


\section{RESULTADOS}

Como fue mencionado en párrafos precedentes, el cálculo de la pérdida de los servicios ambientales considerados en este trabajo mediante el uso de indicadores, demanda el conocimiento de los cambios en los usos del suelo en el período seleccionado. Es por ello que en el primer apartado de los resultados, se incluye dicho análisis para posteriormente arribar a los resultados de los indicadores de sustentabilidad definidos en este trabajo.

\section{Usos del suelo en el partido de Azul (2002 y 2011)}

La clasificación supervisada (Figura 2) permite identificar diferentes usos de suelo para los años 2002 y 2011: urbanos, áreas con pastizales y pasturas, áreas cultivadas (con cultivos anuales), áreas cultivables (combinada por rastrojos recién cosechados y potreros destinados a la siembra próximamente) y agua, para los años 1988 y 2010. Así definidas, las áreas cultivables y cultivadas, representan en conjunto las áreas agrícolas. En la Tabla 2, se presentan las superficies correspondientes a cada una de las clases.

Los usos urbanos incrementaron su superficie en el período considerado, pasando de abarcar el 0,28\% del Partido en 2002 a ocupar el 0,41\% en 2011. Este incremento del $81,6 \%$ se vincula directamente con la expansión de la ciudad de Azul, que conforma un complejo urbano bastante consolidado en materia de servicios, comercio mayorista y minorista, actividades industriales, estructura educativa, entre otros. Según datos los datos censales de 2010, la población del Partido alcanzó los 65.280 habitantes con una densidad de $9,9 \mathrm{hab} . / \mathrm{Km}^{2}$. Esto muestra un incremento del 3,6\% respecto de los datos censales de 2001, año en el cual la población del Partido alcanzó los 62.996 habitantes y una densidad de $9,5 \mathrm{hab} . / \mathrm{Km}^{2}$.

Figura 2. Usos del suelo en el partido de Azul, período 2002- 2011.

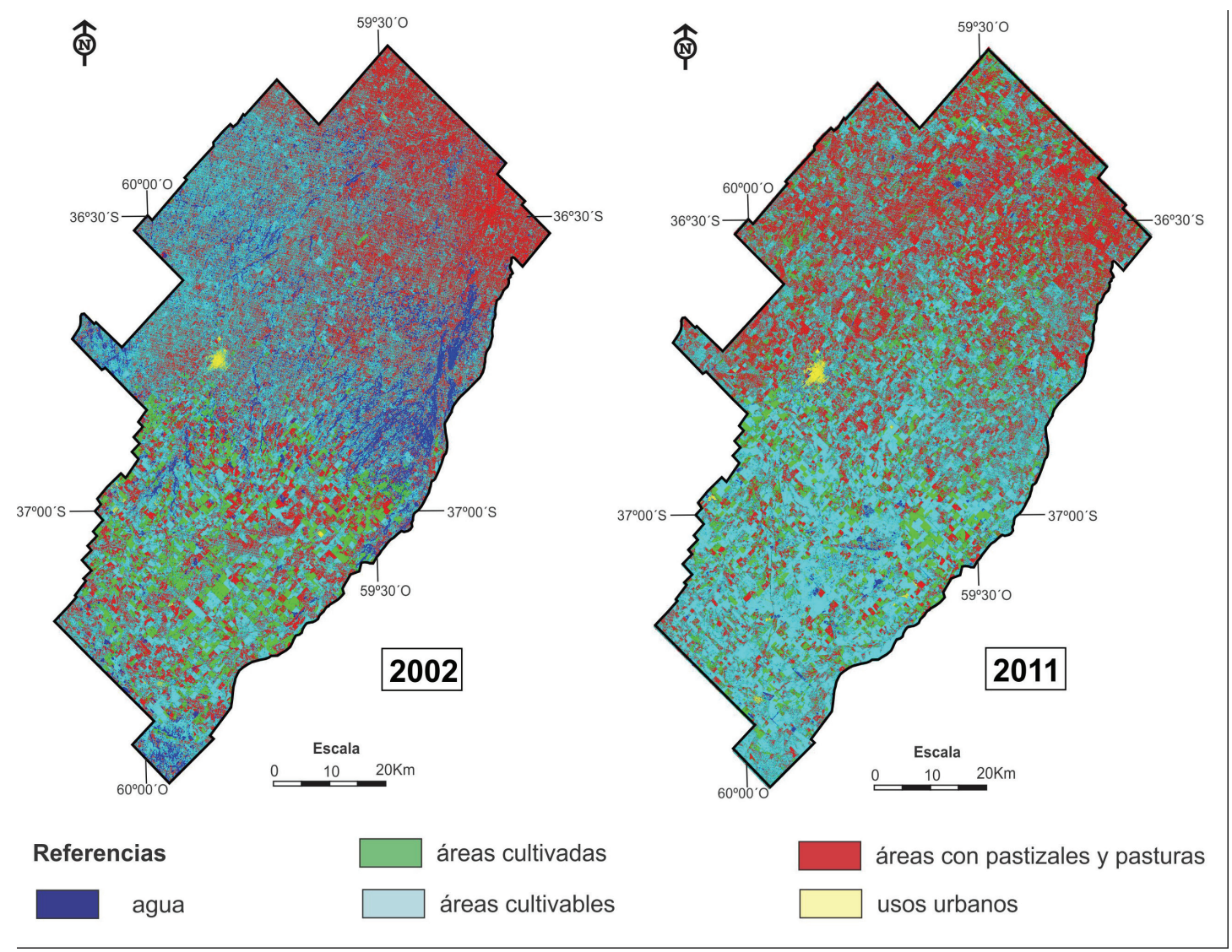

Fuente: Elaboración propia a partir de la clasificación supervisada de las imágenes satelitales (2002-2011). 
Intencificación agrícola y pérdida de servicios ambientales en el partido de Azul (Provincia de Buenos Aires) entre 2002-2011 Patricia Vazquez, Laura Zulaica

Tabla 2. Partido de Azul: superficie ocupada por cada clase, en el período 2002-2011.

\begin{tabular}{|l|c|c|c|c|}
\hline \multicolumn{1}{|c|}{ Clases } & Píxeles 2002 & Superficie 2002 $\left.\mathbf{( K m}^{2}\right)$ & Píxeles 2011 & Superficie 2011 $\left(\mathbf{K m}^{\mathbf{2}}\right)$ \\
\hline Ciudad & 12,43 & 19,10 & 23,25 & 27,09 \\
\hline Agua & 380,51 & 442,87 & 69,12 & 80,50 \\
\hline Áreas con pastizales y pasturas & $2.231,41$ & $2.597,06$ & $2.113,04$ & $2.461,02$ \\
\hline Áreas cultivadas y cultivables & $3.055,32$ & $3.555,97$ & $3.474,25$ & $4.046,39$ \\
\hline Superficie del Partido & $\mathbf{5 . 6 7 9 , 6 7}$ & $\mathbf{6 . 6 1 5 , 0 0}$ & $\mathbf{5 . 6 7 9 , 6 7}$ & $\mathbf{6 . 6 1 5 , 0 0}$ \\
\hline
\end{tabular}

Fuente: Elaboración propia a partir de la clasificación supervisada de las imágenes satelitales de 2002 y 2011.

Con respecto a las áreas con agua, según lo expresado por Usunoff y Varni (1995), el balance hidrológico en el Partido muestra las características propias de los sistemas de llanura, con un neto predominio de los componentes verticales del flujo de agua (evapotranspiración, infiltración) por sobre los horizontales (escurrimiento superficial y subterráneo). De los $900 \mathrm{~mm}$ que, en promedio, precipitan anualmente, el $95 \%$ se evapotranspira, el 2,6\% infiltra (recarga subterránea) y el resto circula por los cursos de agua, principalmente el arroyo Azul. Ante estas condiciones, se observa en las imágenes clasificadas para el período 2002-2011 que las áreas con agua se redujeron significativamente en el período; pasando a ocupar el 6,7\% del Partido en 2002 al 1,21\% en 2011. Esto se debe a que las precipitaciones medias anuales en 2002 fueron de 1.169,5 mm (año húmedo) mientras que en 2011 fueron de 666,5 mm (Centro Regional de Agrometeorología, FAA-UNICEN). Así, la reducción en la superficie destinada a esta clase fue del $81,82 \%$.

El análisis de la imagen de 2002 y sus respectivos estadísticos revela que las áreas con pastizales y pasturas (ganaderas) ocupan el 39,26\% $\left(2.597,06 \mathrm{Km}^{2}\right)$ de la superficie del Partido. La agricultura en este año (áreas cultivables y cultivadas), alcanzaba el 53,75\% de la superficie del Partido $\left(3.555,97 \mathrm{Km}^{2}\right)$. En ese año, existían explotaciones netamente agrícolas, netamente ganaderas, y una importante proporción de mixtas (agrícola-ganaderas). Las áreas agrícolas de las explotaciones superaban en un $14,49 \%$ a las ganaderas.

De acuerdo con lo expresado por Requesens (2005) en un análisis efectuado a partir de los datos de los Censos Nacionales Agropecuarios de 1988 y 2002, se manifiesta en el período una tendencia a la expansión de áreas agrícolas. En ese sentido, el autor destaca que la superficie implantada con cultivos anuales aumentó un $66 \%$, mientras que la superficie implantada con pasturas perennes apenas tuvo un incremento inferior al 10\% en el Partido.

En 2011, las áreas con pastizales naturales y pasturas, ocupan un $37,20 \%$ de la superficie del Partido $\left(2.461,02 \mathrm{Km}^{2}\right)$, mientras que las agrícolas alcanzan $61,16 \%\left(4.046,39 \mathrm{Km}^{2}\right)$. Esto significa que las últimas áreas mencionadas muestran un incremento respecto de 2002. Este hecho se evidencia en la conversión de establecimientos mixtos a netamente agrícolas, favorecidos fundamentalmente por la mejor inserción de estos cultivos en los mercados internacionales. De acuerdo con la información obtenida en campo, este incremento de las áreas agrícolas, tuvo lugar especialmente a partir de 2007. Los datos aportados por Requesens (2010) obtenidos de la Secretaría de Agricultura, ganadería, Pesca y Alimentación (SAGPyA), indican una especie de meseta en proceso de agriculturización entre 2002 y 2007, que tendería a revertirse a partir del año mencionado.

Con la información obtenida a partir del análisis de las imágenes y el trabajo de campo, es posible estimar la pérdida de los servicios ambientales considerados en este trabajo.

\section{Pérdida de servicios ambientales en el partido de Azul}

Ahora bien, como fue mencionado en la metodología, para la estimación de la pérdida de servicios ambientales se utilizaron indicadores de sustentabilidad que en este caso, permite dimensionar la conservación de la calidad de suelos y agua, en este caso asociada directamente con el uso de plaguicidas, y la conservación de la biodiversidad.

Es importante destacar que el valor absoluto de los indicadores no tienen significado en sí mismo, sino que su utilidad reside en la capacidad de comparar, en este caso, la pérdida de servicios ambientales en el Partido en distintos años. 


\section{Indicador de Conservación de la calidad de suelos $y$ aguas (CCSA)}

Como fue mencionado, este servicio ambiental se asocia en este caso con el uso de agroquímicos. Para poder estimar la pérdida del servicio fue necesario realizar entrevistas a fin de conocer los principales agroquímicos utilizados en los establecimientos agrícolas en 2002 y 2011 y la cantidad aplicada por unidad de superficie.
Los resultados revelan que muchos de los principios activos de los compuestos químicos aplicados el período son esencialmente los mismos, lo que varía es la marca comercial y los valores de venta de los productos. Asimismo, cambia la cantidad aplicada por unidad de superficie en el año y la superficie en la que se emplean. En la Tabla 3 se muestran los principales plaguicidas empleados en las áreas de cultivo en 2002 y 2011, con los parámetros a evaluar en el indicador de CCSA.

Tabla 3. Conservación de la calidad de suelos y aguas en el partido de Azul (2002 y 2011).

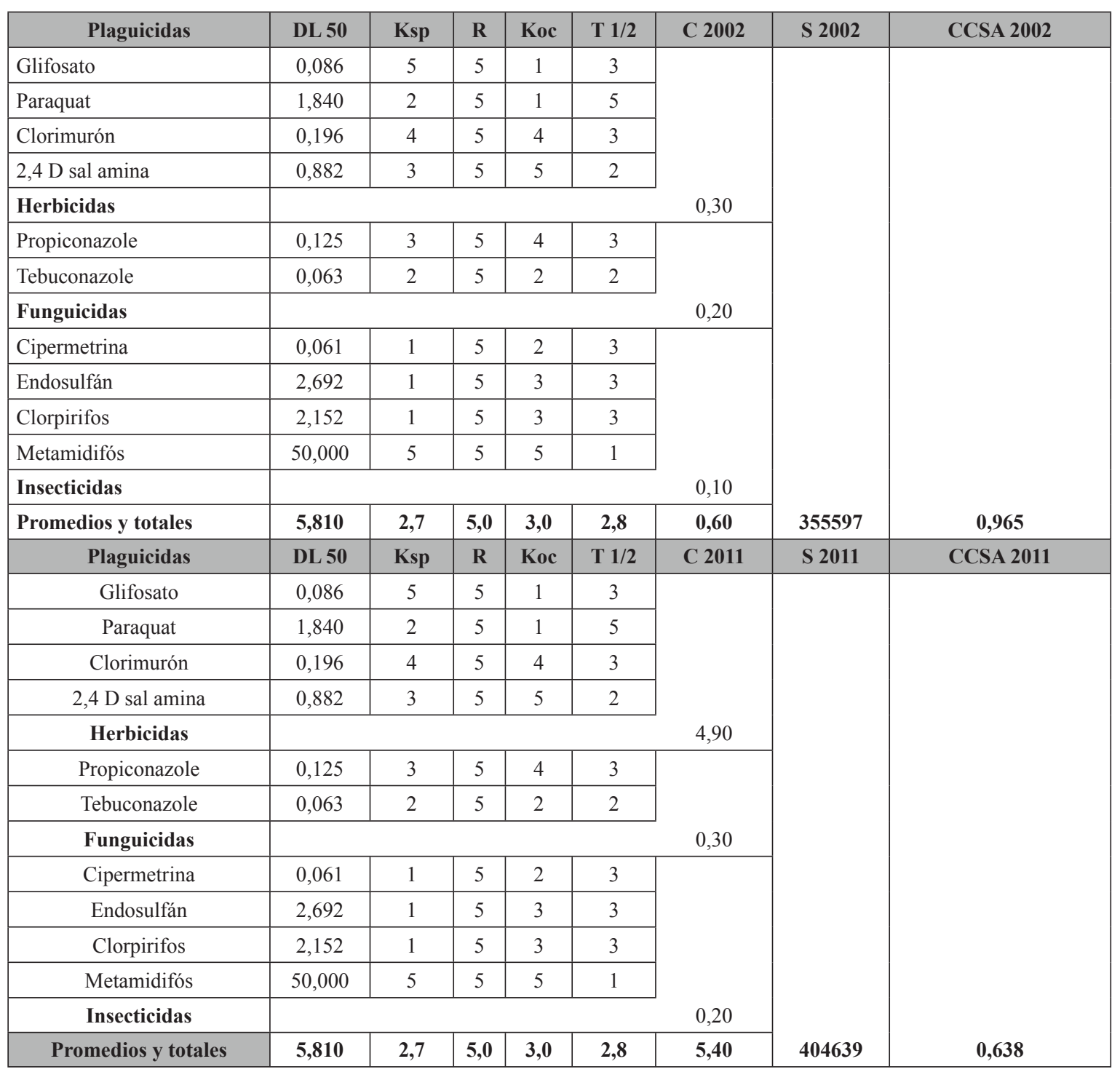

DL 50: dosis letal 50 (ton/g); Ksp: solubilidad (g/g); R: permeabilidad ( 5 corresponde al valor establecido para la Pampa Deprimida); Koc: coeficiente de adsorción del compuesto por la fase orgánica del suelo (g/g); T12/2: vida media de los productos utilizados (días); C: cantidad de producto aplicada anualmente por hectárea (1/ha); S: superficie de áreas agrícolas en el Partido (ha); y CCSA: Conservación de la calidad de suelos y agua en el año señalado.

Fuente: Elaboración propia sobre la base de Viglizzo (2003), Vazquez y Zulaica (2012); Vazquez et al. (2012) y entrevistas. 
Siguiendo el procedimiento especificado en la metodología, el indicador de CCSA para el año 2002 en el partido de Azul revela un valor de 0,965, mientras que en 2011 desciende a 0,638. Esta disminución del $934,29 \%$ en dicho período se justifica fundamentalmente por el cambio en las prácticas productivas (Viglizzo et al., 2002). Satorre (2005) expresa que en las últimas décadas, la agricultura de nuestro país en general, y de la región pampeana en particular, ha experimentado una notable expansión a partir de un desarrollo tecnológico basado en cultivos transgénicos, siembra directa, incremento en el uso de fertilizantes y plaguicidas y, más recientemente, agricultura de precisión. El cambio tecnológico y la expansión de la superficie cultivada han sido liderados por el cultivo de soja en base al desarrollo de variedades transgénicas resistentes a glifosato, lo que facilitó la rápida expansión y el aumento de rendimiento de este cultivo (MARTINEZ-GHERSA y GHERSA, 2005).

Lo anterior, se condice con lo que plantea Pimentel (1992), quien expresa que durante los últimos 50 años, los pesticidas (insecticidas, herbicidas $\mathrm{y}$ fungicidas) se han convertido en uno de los componentes principales de la agricultura y, aunque su uso es considerado económicamente rentable para la mayoría de los sistemas, los efectos secundarios que tienen sobre el ambiente son frecuentemente negativos. Lo mencionado conlleva a plantearse la urgente necesidad de lograr un uso responsable de los pesticidas, siendo esto una prioridad fundamental en la agenda de la investigación agrícola.

El actual modelo agroproductivo, demanda la aplicación sostenida de plaguicidas, generalmente de segunda generación, de baja a moderada persistencia. Esta nueva modalidad productiva permite ampliar la superficie real a sembrar, incrementando la cantidad de productos fitosanitarios aplicada por unidad de superficie en el año. De estos productos se genera sobre todo un aumento exponencial en los herbicidas. Así, de acuerdo con los valores obtenidos en el cálculo del indicador, la pérdida del servicio ambiental de conservación de la calidad de suelos y agua ligada al uso de agroquímicos fue de $33,8 \%$ entre 2002 y 2011.

A fin de mostrar sintéticamente la incidencia del uso de plaguicidas sobre la conservación de suelos y agua, se presenta la Tabla 4, en la cual se describen sus principales efectos, según Linares (2007). Las consecuencias no son menores, dado que, la contaminación generada incide directamente sobre la calidad de los suelos y recursos hídricos superficiales y subterráneos, principal fuente de abastecimiento en el área.

Tabla 4. Efectos de los plaguicidas suelos y agua.

\begin{tabular}{|l|l|l|}
\hline \multicolumn{1}{|c|}{ Tipos de plaguicidas } & \multicolumn{1}{|c|}{ Localización } & \multicolumn{1}{c|}{ Efectos } \\
\hline Herbicidas & suelos & Una sobrecarga de residuos afecta las cosechas posteriores. \\
\cline { 2 - 3 } & agua & Mata o inhibe la acción de algunas plantas acuáticas. \\
\hline \multirow{2}{*}{$\begin{array}{l}\text { Ácidos aromáticos } \\
\text { ésteres, carbamatos }\end{array}$} & suelos & Su persistencia puede afectar cosechas posteriores. \\
\cline { 2 - 3 } & agua & $\begin{array}{l}\text { La erosión superficial puede transportar herbicidas a los sistemas acu- } \\
\text { áticos. }\end{array}$ \\
\hline Insecticidas-Fungicidas & \multicolumn{2}{|l}{$\begin{array}{l}\text { Los residuos afectan las cosechas posteriores, el transporte por las aguas } \\
\text { superficiales afecta las plantas acuáticas. }\end{array}$} \\
\hline \multirow{2}{*}{\begin{tabular}{l} 
Organoclorados \\
\cline { 2 - 3 }
\end{tabular}} & suelos & $\begin{array}{l}\text { Las aguas contaminadas pueden afectar las plantas si se usan para irri- } \\
\text { gación. }\end{array}$ \\
\cline { 2 - 3 } $\begin{array}{l}\text { Organofosforados, carba- } \\
\text { matos, } \\
\text { piretroides }\end{array}$ & suelos & Tienen corta vida media, por lo que sus efectos sobre las plantas es escaso. \\
\cline { 2 - 3 } & agua & Tóxicos para ciertas algas. \\
\hline
\end{tabular}

Fuente: Linares (2007). 


\section{Indicador de Conservación de la biodiversidad (CB)}

El proceso de agriculturización conlleva a una simplificación estructural y funcional de los ecosistemas. A fin de dimensionar el impacto de los cambios de uso sobre el hábitat original y la biodiversidad del Partido, se utilizó el indicador CB. Dicho indicador fue calculado para 2002 y 2011, siguiendo el procedimiento detallado en la metodología. En la Tabla 5, se especifican los coeficientes aplicados para cada año y los resultados obtenidos para ambos años.

Cuando se incorporan los datos relativos a la superficie de las actividades desarrolladas en el partido de Azul, se observa que disminuye su biodiversidad. Así, puede verificarse que entre 2002 y 2011 existe una disminución en el valor del indicador asociado con el servicio $C B$, el cual desciende de 0,343 a 0,288. El cambio de uso, se tradujo en una disminución del 16,03\% en el servicio conservación de la biodiversidad evaluado según el indicador, entre los años considerados.

Los resultados obtenidos se condicen con lo que expresado por Hooper et al. (2005) y Schröter et al. (2005) quienes plantean que los problemas ambientales generados por los aumentos de la expansión agrícola y la intensificación de la agricultura se incrementaron, produciendo alteraciones a partir del reemplazo de bosques, pastizales y humedales por sitios agrícolas. Esto genera alteraciones en la biodiversidad global en todos los niveles, es decir, desde la diversidad genética dentro de poblaciones hasta la diversidad de ecosistemas en un paisaje, ya sea alterando la composición, riqueza y equitatividad, como las interacciones entre organismos, y dispersión de la biota más allá de los límites geográficos naturales.

Tabla 5. Partido de Azul: Conservación de la biodiversidad en 2002 y 2011.

\begin{tabular}{|c|c|c|c|c|c|c|c|c|c|}
\hline & Actividades & cpSupAc & $\mathrm{Ce}$ & Or & Pe & Oev & Osv & RPIH & CB \\
\hline \multirow{4}{*}{ స్ర్స } & Agrícolas & 0,538 & 10 & 7,5 & 5 & 0 & 0 & 0,865 & 0,465 \\
\hline & Ganaderas & 0,393 & 5 & 7,5 & 0 & 0 & 0 & 0,481 & 0,189 \\
\hline & Urbanas & 0,003 & 10 & 7,5 & 5 & 2,5 & 1 & 1,000 & 0,003 \\
\hline & Total & & & & & & & & 0,343 \\
\hline \multirow{4}{*}{ ב্ন } & Agrícolas & 0,612 & 10 & 7,5 & 5 & 0 & 0 & 0,865 & 0,529 \\
\hline & Ganaderas & 0,372 & 5 & 7,5 & 0 & 0 & 0 & 0,481 & 0,179 \\
\hline & Urbanas & 0,004 & 10 & 7,5 & 5 & 2,5 & 1 & 1,000 & 0,004 \\
\hline & Total & & & & & & & & 0,288 \\
\hline
\end{tabular}

cpSupAc: coeficiente de ponderación que indica la proporción de la superficie del Partido ocupada por la actividad; Ce: coeficiente relativo a la cantidad de especies; Or: coeficiente referido al origen; Pe: coeficiente de periodicidad; Oev: coeficiente de organización de estratos verticales; y Osv: coeficiente de estratos subverticales; RPIH: indicador de riesgo parcial de intervención del hábitat;

CB: indicador de conservación de la biodiversidad.

Fuente: Elaboración propia sobre la base de Viglizzo (2003), Vazquez y Zulaica (2012); Vazquez et al. (2012) y entrevistas. 
Sin duda, los cambios en el uso del suelo se traducen en la pérdidas de servicios ambientales importantes referidos al hábitat y la reserva genética (SILVAARROYAVE y CORREA RESTREPO, 2009), estimados por el indicador.

\section{DISCUSIÓN FINAL Y CONCLUSIONES}

Los resultados obtenidos indican que en el período 2002-2011, se generó una intensificación agrícola con la implementación del doble cultivo anual. Asimismo, la expansión de la agricultura (13,79\%), se produjo a expensas de la disminución de la ganadería, en un 5,24\%, y de la superficie de agua que disminuyó un $81,82 \%$ entre 2002 y 2011. La agriculturización se manifiesta no sólo en las áreas bien drenadas de las llanuras periserranas, sino también en las tierras achatadas y anegables de las planicies deprimidas, las cuales ocupan alrededor del $70 \%$ de la superficie del Partido.

Dichos cambios agroproductivos se reflejan en la pérdida de servicios ambientales de regulación y soporte como son la conservación de la calidad de suelos y agua y la conservación de la biodiversidad y se traduce en los resultados obtenidos en la estimación de los indicadores de sustentabilidad.

En ausencia de conocimientos y políticas adecuadas, las últimas décadas no sólo han dado lugar a pérdidas severas en la capacidad de los ecosistemas para sostener la productividad de los suelos, proveer agua limpia, controlar los caudales de ríos e inundaciones, o para regular la composición de la atmósfera y el clima (entre otros beneficios); también han dado lugar a una asimetría marcada en la forma en que el perjuicio de esas pérdidas se distribuye entre distintos sectores geográficos y económicos de la sociedad (LATERRA et al., 2011). De acuerdo con el autor citado, el progresivo reconocimiento científico y el mayor nivel de conciencia sobre los múltiples beneficios, tanto tangibles como intangibles, que brindan los ecosistemas, ha vuelto inadmisible el reemplazo indiscriminado de bosques, pastizales y humedales remanentes para su aprovechamiento agrícola, forestal, pecuario, minero o inmobiliario sin una adecuada evaluación de los efectos colaterales o externalidades que los acompañan.
Así, la falta de planificación en el uso de los recursos naturales y de tecnologías y políticas apropiadas que garanticen su preservación, ha provocado un agudo deterioro ambiental en la región, que se traduce en pérdida de biodiversidad, y en degradación de los suelos, disminución de la disponibilidad de agua dulce, cambio de los cauces de los ríos por azolvamiento y disminución de la calidad de sus aguas por contaminación y sedimentación (CEPAL, 2002).

Lamentablemente, la valoración de los servicios ambientales mencionados no se ha extendido a todos los ciudadanos del planeta, ni a los gobiernos, en el sentido de la urgencia que requieren las acciones destinadas a frenar y revertir los fuertes impactos que la sociedad está produciendo desde hace varias décadas sobre los ecosistemas naturales.

Ante los problemas enunciados, se considera prioritario tomar conciencia acerca de que el valor de los ecosistemas va mucho más allá del valor económico directo. Los servicios que prestan son indispensables para la supervivencia de la humanidad en el planeta: permiten la estabilización climática y atmosférica; regulan el ciclo hídrico y de la humedad mesoclimática; son fuente de productos forestales maderables, de la vida silvestre, farmacéuticos, entre muchos otros; y representan un valor paisajístico y turístico creciente.

\section{REFERENCIAS}

BURKART, R.; BÁRBARO, N. O.; SÁNCHEZ, R. O.; GÓMEZ, D. A. Eco-regiones de la Argentina. Buenos Aires: Secretaría de Recursos Naturales y Desarrollo Sustentable, 1999.

CABRERA, A.; WILLINK, A. Biogeografía de América Latina. Washington: Secretaría General de la Organización de los Estados Americanos, 1973.

CARREÑO, L.; VIGLIZZO, E. Provisión de los servicios ecológicos y gestión de los ambientes rurales en Argentina. Buenos Aires: INTA, Área estratégica de gestión ambiental, 2011. 
CEPAL. La sostenibilidad del desarrollo en América Latina y el Caribe: desafios y oportunidades. Santiago de Chile: CEPAL-Oficina Regional del PNUMA para América Latina y el Caribe, 2002.

CHUVIECO, E. Teledetección Ambiental. La observación de la tierra desde el espacio. Barcelona: Editorial Ariel Ciencia, 2007.

DAILY, G. C.; SÖDERQVIST, S.; ANIYAR, K.; ARROW, P. The value of nature and the nature of value. Science. 289: 395-396, 2000.

HOOPER, D.; CHAPIN, III S.; EWEL, J.; HECTOR, A.; INCHAUSTI, P. Effects of biodiversity on ecosystem functioning: a consensus of current knowledge. Ecological Monographs. v.75, n.1, 2005.

INDEC. Censo Pacional de Población, Hogares y Viviendas. Buenos Aires: Instituto Nacional de Estadísticas y Censos, 2010.

MANUEL-NAVARRETE, D; GALLOPÍN, G. Integración de politicas, sostenibilidad y agriculturización en la pampa argentina y áreas extrapampeanas. Santiago de Chile: CEPAL. Serie Seminarios y Conferencias $n^{\circ} 50,2007$.

MARTINEZ-GHERSA, M. A.; GHERSA, C. M. Consecuencias de los recientes cambios agrícolas. Ciencia Hoy, 15: 37-45, 2005.

LATERRA, P.; JOBBAGY E. G.; PARUELO J. Valoración de servicios ecosistémicos; conceptos, herramientas y aplicaciones para el ordenamiento territorial. Buenos Aires: INTA, 2011.

LINARES, R. M. Evaluación ambiental de pesticidas organoclorados en sedimentos de la laguna de Chantuto (Chiapas, México) y de la bahía de Santander (Cantabria, España). Tesis doctoral, Universidad de Cantabria, Ingeniería Química, 2007.

MEA (Millennium Ecosystem Assessment) Ecosystems and Human Well-Being. A Framework for Assessment. Washington: Island Press, 2003.
MEA (Millennium Ecosystem Assessment). Living beyond Our Means: Natural Assets and Human Well-Being. Washington: Island Press, 2005.

PIMENTEL, D. Ecological effects of pesticides of Non-Target species in terrestrial ecosystems. In: TARDIFF, R. G. (org.) Methods to Assess Adverse Effects of Pesticides on Non-target Organisms. New York: John Wiley \& Sons, 1992.

REQUESENS, E. Ambiente, uso de la tierra y agrodiversidad en el paisaje serrano y periserrano del Partido de Azul. In: $66^{\circ}$ CONGRESO NACIONAL DE GEOGRAFÍA. Actas... Buenos Aires, p. 257-264, 2005.

REQUESENS, E. Perspectivas mundiales, nacionales y regionales de la producción de cereales y carne. Revista de la Sociedad Rural de Azul, p. 7-10, 2010.

RUEDA, S. Modelos e indicadores para ciudades más sostenibles. Cataluña: Fundació Fòrum Ambiental, 1999.

SATORRE, E. Cambios tecnológicos en la agricultura argentina actual. Ciencia Hoy. 15, 2005.

SÁNCHEZ, R.; MATTUS, G.; ZULAICA, L., Compartimentación ecológica y ambiental del partido de Tandil (Provincia de Buenos Aires). In: CONGRESO AMBIENTAL '99. Actas... Programa de Estudios Ambientales, Universidad Nacional de San Juan, San Juan, 1999.

SCHRÖTER, D.; CRAMER, W.; LEEMAN, R.; PRENTICE, C.; ARAUJO, M. Ecosystem service supply and vulnerability to global change in Europe. Science. 310. 2005.

SEMARNAT. Introducción a los servicios ambientales. México: Secretaría de Medio Ambiente y Recursos Naturales (SEMARNAT), 2003.

SILVAARROYAVE, S. M.; CORREA RESTREPO, F. J. Análisis de la contaminación del suelo: revisión de la normativa y posibilidades de la regulación económica. Semestre Económico, v. 12, n. 23, p. 13-34, 2009. 
SOLBRIG, O.; VIGLIZZO, E. Sustainable Farming in the Argentine Pampas: History, Society, Economy and Ecology. DRCLAS Working Paper n. 99/00-1. Cambridge: Universidad de Harvard, 1999.

USUNOFF, E., VARNI M. Hidrología de los sectores alto y medio de la cuenca del arroyo Azul. Informe final. Azul: Instituto de Hidrología de Llanuras, 1995.

VARSAVSKY, A. I.; FERNÁNDEZ DILLON, D. Indicadores de sustentabilidad. Se utilizan correctamente? In: $13^{\circ} \mathrm{CONGRESO} \mathrm{ARGENTINO}$ DE SANEAMIENTO Y MEDIO AMBIENTE. Buenos Aires, 2003. Actas... AIDIS, Buenos Aires, 2003.

VAZQUEZ, P.; ZULAICA, L. Transformaciones agroproductivas e indicadores de sustentabilidad en el Partido de Tandil (Provincia de Buenos Aires) durante los períodos 1988-2002 y 20022010. Revista Campo-Território. Uberlândia, v.7, n.13, 2012.

VAZQUEZ, P.; SACIDO, M.; ZULAICA, L. Transformaciones agroproductivas e indicadores de sustentabilidad en la Cuenca del río Quequén Grande, Provincia de Buenos Aires, Argentina) durante los períodos 1988-1998 y 1998-2008. Cuadernos Geográficos, № 50, 2012.
VIGLIZZO, E. Manual AGRO-ECO-INDEX. Programa Nacional de Gestión Ambiental Agropecuaria-Proyecto de Eco-Certificación. Buenos Aires: INTA, 2003.

VIGLIZZO, E. Agricultura, clima y ambiente en Argentina: tendencias, interacciones e impacto. In: SOLBRIG, O. T. y. ADÁMOLI (org.) Agro $y$ ambiente: una agenda compartida para el desarrollo sustentable. Buenos Aires: Foro J de la Cadena Agroindustrial Argentina, 2008.

VIGLIZZO, E. F.; PORDOMINGO, A. J.; CASTRO, M. G.; LÉRTORA, F. A. La sustentabilidad ambiental de la agricultura pampeana ¿oportunidad o pesadilla? Ciencia Hoy, v. 12, n. 8, p. 38-51, 2002.

VIGLIZZO, E. F.; PORDOMINGO, A. J.; CASTRO, M. G.; LÉRTORA, F. A. Environmental assessment of agriculture at a regional scale in the pampas of Argentina. Environmental Monitoring and Assessment. Center for Research on Sustainable Forests, University of Maine, n.87, p.169-195, 2003. 